\title{
THE STRUCTURE OF THE MINIMAL FREE RESOLUTION OF SEMIGROUP RINGS OBTAINED BY GLUING
}

\author{
PHILIPPE GIMENEZ AND HEMA SRINIVASAN
}

\begin{abstract}
We construct a minimal free resolution of the semigroup ring $k[C]$ in terms of minimal resolutions of $k[A]$ and $k[B]$ when $\langle C\rangle$ is a numerical semigroup obtained by gluing two numerical semigroups $\langle A\rangle$ and $\langle B\rangle$. Using our explicit construction, we compute the Betti numbers, graded Betti numbers, regularity and Hilbert series of $k[C]$, and prove that the minimal free resolution of $k[C]$ has a differential graded algebra structure provided the resolutions of $k[A]$ and $k[B]$ possess them. We discuss the consequences of our results in small embedding dimensions. Finally, we give an extension of our main result to semigroups in $\mathbb{N}^{n}$.
\end{abstract}

\section{INTRODUCTION}

Given a subset of positive integers $C=\left\{c_{1}, \ldots, c_{n}\right\}$, we denote by $\langle C\rangle$ the semigroup generated by $C$. When $\operatorname{gcd}\left(c_{1}, \ldots, c_{n}\right)=1$, i.e., when $\mathbb{N} \backslash\langle C\rangle$ is a finite set, we call $\langle C\rangle$ a numerical semigroup. We say $C$ minimally generates the semigroup $\langle C\rangle$ if no proper subset of $C$ generates the same semigroup $\langle C\rangle$. The minimal generating set of a semigroup is unique.

Let $k$ be an arbitrary field. Consider the algebra homomorphism $\phi_{C}: k\left[x_{1}, \ldots, x_{n}\right] \rightarrow$ $k[t]$ induced by $\phi_{C}\left(x_{i}\right)=t^{c_{i}}$. The semigroup ring $k[C]=k\left[t^{c_{i}} \mid 1 \leq i \leq n\right]$ is the image of $\phi_{C}$ and it is isomorphic to $k\left[x_{1}, \ldots, x_{n}\right] / I_{C}$ where $I_{C}=\operatorname{ker} \phi_{C}$ is the prime ideal defining the affine monomial curve $\mathfrak{C}_{C} \subset \mathbb{A}_{k}^{n}$ whose coordinate ring is $k[C]$. The ideal $I_{C}$ is binomial and it is homogeneous if one gives to each variable $x_{i}$ weight $c_{i}$. Hence, one can consider graded free resolutions of $k[C]$ over the polynomial ring graded this way. If $\langle C\rangle$ is minimally generated by $C$, we say that the embedding dimension of $\langle C\rangle$ is $n$. The ring $k[C]$ has Krull dimension 1 and hence it is always Cohen-Macaulay. Observe that if one studies the semigroup ring $k[C]$, one can always assume without loss of generality that $\operatorname{gcd}\left(c_{1}, \ldots, c_{n}\right)=1$ since one gets the same ideal $I_{C}$ when one divides all the integers in $C$ by their gcd. When the ideal $I_{C}$ is generated by a regular sequence, we say the numerical semigroup $\langle C\rangle$, the semigroup ring $k[C]$, and the monomial curve $\mathfrak{C}_{C}$, are complete intersections. Similarly, we say the semigroup $\langle C\rangle$ and the monomial curve $\mathfrak{C}_{C}$ are Gorenstein when the semigroup ring $k[C]$ satisfies this property. Note that the fact

The first author was partially supported by Ministerio de Ciencia e Innovación (Spain), MTM201678881-P.

Keywords: semigroup rings, numerical semigroups, gluing, free resolutions, Betti numbers.

$2000 \mathrm{MSC}: 13 \mathrm{D} 02,20 \mathrm{M} 25,13 \mathrm{~A} 02,20 \mathrm{M} 14$. 
that $k[C]$ is Gorenstein or a complete intersection does not depend on the field $k$ and that's why we can talk about Gorenstein and complete intersection semigroups.

Numerical semigroups of embedding dimension 1 are isomorphic to the integers and hence their semigroup ring is simply the polynomial ring $k[x]$. When the embedding dimension is 2 , the semigroup ring $k[C] \simeq k[x, y] /\left(x^{a}-y^{b}\right)$ is the coordinate ring of a hypersurface. When the embedding dimension is three, $k[C] \simeq k[x, y, z] / I_{C}$ is either a complete intersection or $I_{C}$ is the ideal of the $2 \times 2$ minors of a $2 \times 3$ matrix of the form $\left(\begin{array}{lll}x^{a} & y^{b} & z^{c} \\ y^{d} & z^{e} & x^{f}\end{array}\right)$ by [14, Theorems 3.7 and 3.8]. When the embedding dimension is four or more, there is no such simple classification for the semigroup rings. In fact, when $C=\left\{c_{1}, c_{2}, c_{3}, c_{4}\right\}$, there is no upper bound for the minimal number of generators for the ideal $I_{C},[4]$.

In this paper, we construct a minimal graded free resolution for the semigroup ring $k[C]$ when $\langle C\rangle$ is a numerical semigroup obtained by gluing two smaller numerical semigroups. This is our main Theorem 3.1 .

Our paper is organized as follows. In section 2, we recall the definition of semigroups obtained by gluing and give some of their properties, known and new. Section [3] has our main result and the consequences. For numerical semigroups obtained by gluing, we compute the invariants associated to the minimal graded free resolution like the Betti numbers (Corollary 3.2), the graded Betti numbers (Proposition [3.6), the CastelnuovoMumford regularity (Corollary 3.7) and the Hilbert series (Corollary 3.8). In particular, we show that if $\langle C\rangle$ is obtained by gluing $\langle A\rangle$ and $\langle B\rangle$, then the Cohen-Macaulay type of $k[C]$ is the product of the Cohen-Macaulay types of $k[A]$ and $k[B]$ (Corollary 3.3). We also show that the minimal resolution of $k[C]$ has a differential graded algebra structure provided the minimal resolutions of $k[A]$ and $k[B]$ do (Proposition 3.9). The multiplication on the resolution of $k[C]$ is explicitly written from those of $k[A]$ and $k[B]$. In section 4, we consider the case when the numerical semigroup $\langle C\rangle$ decomposes as $C=k_{1} A \sqcup k_{2} B$ where one of the subset of $C$ is a singleton. In section 5, we discuss the consequences of our results for small embedding dimensions and give some examples. In particular, we classify decomposable numerical semigroups up to embedding dimension 5 (Propositions 5.1 and 5.4) and also show that, in embedding dimension $n \geq 4$, there exist indecomposable numerical semigroups of any Cohen-Macaulay type between 1 and $n$ (Corollary 5.3). Finally, in the last section we observe that our main result is valid for decomposable semigroups in higher dimension (Theorem 6.1) and give an example to illustrate this.

\section{Numerical SEMigroups obtained By GLuing}

2.1. Definition. A numerical semigroup $\langle C\rangle$ is obtained by gluing two semigroups $\langle A\rangle$ and $\langle B\rangle$ if its minimal generating set $C$ can be written as the disjoint union of two subsets, $C=k_{1} A \sqcup k_{2} B$, where $A$ and $B$ are minimal generators of numerical semigroups $\langle A\rangle$ and $\langle B\rangle$, and $k_{1}, k_{2}$ are relatively prime positive integers such that $k_{1}$ is in the numerical 
semigroup $\langle B\rangle$ but not in $B$ and $k_{2} \in\langle A\rangle \backslash A$. When this occurs, we also say that $\langle C\rangle$ is decomposable, or that $\langle C\rangle$ is a gluing of $\langle A\rangle$ and $\langle B\rangle$.

This way to decompose the minimal generating set of a numerical semigroup already appears in the classical paper by Delorme [9] where it is used to characterize complete intersection numerical semigroups. In [16], Rosales introduces the concept of gluing for finitely generated subsemigroups of $\mathbb{N}^{n}$ in terms of a presentation for the semigroup, and he shows that, for numerical semigroups, his definition coincides with the decomposition in 9 ; see section 2.3 below.

Note that one can extend the above definition when $k_{1}$ and $k_{2}$ are not relatively prime. If the positive integers in $C$ are not relatively prime and $d$ is their gcd, then the semigroup $\langle C\rangle$ is decomposable if and only if the numerical semigroup $\left\langle\frac{C}{d}\right\rangle$ is decomposable. In other words, if $C=k_{1} A \sqcup k_{2} B$ and $k_{i}=d k_{i}^{*}$ for $i=1,2$ with $d=\operatorname{gcd}\left(k_{1}, k_{2}\right)$, we ask that $k_{1}^{*} \in\langle B\rangle \backslash B$ and $k_{2}^{*} \in\langle A\rangle \backslash A$ to say that $\langle C\rangle$ is a gluing of $\langle A\rangle$ and $\langle B\rangle$.

2.2. Notations. When $C=k_{1} A \cup k_{2} B$ with $k_{1} \in\langle B\rangle$ and $k_{2} \in\langle A\rangle$, in particular if $\langle C\rangle$ is obtained by gluing $\langle A\rangle$ and $\langle B\rangle$, we will use the following notations:

- $p=\# A, q=\# B$, and the elements in $A$ and $B$ are $\left\{a_{1}, \ldots, a_{p}\right\}$ and $\left\{b_{1}, \ldots, b_{q}\right\}$.

- We consider the three polynomial rings $R_{A}=k\left[x_{1}, \ldots, x_{p}\right], R_{B}=k\left[y_{1}, \ldots, y_{q}\right]$ and $R=k\left[x_{1}, \ldots, x_{p}, y_{1}, \ldots, y_{q}\right]$. Then, the semigroup ring $k[A]$ is isomorphic to $R_{A} / I_{A}$ where $I_{A}$ is the kernel of the algebra homomorphism $\phi_{A}: R_{A} \rightarrow k[t]$ induced by $\phi_{A}\left(x_{i}\right)=t^{a_{i}}$. Similarly, $k[B] \simeq R_{B} / I_{B}$ and $k[C] \simeq R / I_{C}$.

- $F_{A}$ and $F_{B}$ are minimal graded free resolutions for $k[A]$ and $k[B]$ as modules over $R_{A}$ and $R_{B}$ respectively, where variables $x_{i}$ and $y_{j}$ in the polynomial rings $R_{A}$ and $R_{B}$ are given weight $a_{i}$ and $b_{j}$ to make the modules $k[A]$ and $k[B]$ graded. When needed, the differentials will be denoted by $\partial_{i}^{A}:\left(F_{A}\right)_{i} \rightarrow\left(F_{A}\right)_{i-1}$ and $\partial_{j}^{B}:\left(F_{B}\right)_{j} \rightarrow\left(F_{B}\right)_{j-1}$ for $1 \leq i \leq p-1$ and $1 \leq j \leq q-1$. By definition, the resolutions $F_{A}$ and $F_{B}$ are minimal if and only if $\partial_{i}^{A}\left(\left(F_{A}\right)_{i}\right) \subset\left(x_{1}, \ldots, x_{p}\right)\left(F_{A}\right)_{i-1}$ and $\partial_{j}^{B}\left(\left(F_{B}\right)_{j}\right) \subset\left(y_{1}, \ldots, y_{q}\right)\left(F_{B}\right)_{j-1}$ for all $1 \leq i \leq p-1$ and $1 \leq j \leq q-1$.

- Since $k_{1} \in\langle B\rangle$ and $k_{2} \in\langle A\rangle$, there exist non negative integers $\alpha_{i}, \beta_{i}$ such that $k_{1}=\sum_{j=1}^{q} \beta_{j} b_{j}$ and $k_{2}=\sum_{i=1}^{p} \alpha_{i} a_{i}$, and we consider the binomial $\rho=\prod_{i=1}^{p} x_{i}^{\alpha_{i}}-$ $\prod_{j=1}^{q} y_{j}^{\beta_{j}} \in R$. It is homogeneous of degree $k_{1} k_{2}$ if one gives to each variable in $R$ the corresponding weight in $C=\left\{k_{1} a_{1}, \ldots, k_{1} a_{p}, k_{2} b_{1}, \ldots, k_{2} b_{q}\right\}$.

2.3. Properties. The following lemma helps to understand why, in the definition of gluing, we consider $k_{1} \notin B$ and $k_{2} \notin A$. It is slightly more precise than [9, Prop. 10 (ii)].

Lemma 2.1. If $A$ and $B$ minimally generate $\langle A\rangle$ and $\langle B\rangle$ respectively, and $k_{1} \in\langle B\rangle$ and $k_{2} \in\langle A\rangle$ are two relatively prime positive integers, then $C=k_{1} A \cup k_{2} B$ is a disjoint union and minimally generates the semigroup $\langle C\rangle$ if and only if $k_{1} \notin B$ and $k_{2} \notin A$.

Proof. First observe that the assumption in the lemma is necessary: if $k_{1} \in B$ or $k_{2} \in A$ then $k_{1} k_{2} \in C$ and it is not a minimal generator of $\langle C\rangle$ unless $k_{1} \in B$ and $k_{2} \in A$, but in this case $k_{1} k_{2} \in k_{1} A$ and $k_{1} k_{2} \in k_{2} B$ and hence the union $k_{1} A \cup k_{2} B$ is not disjoint. 
Now assume that $k_{1} \notin B$ and $k_{2} \notin A$. If $C$ is not a minimal generating set of $\langle C\rangle$ or if the union $C=k_{1} A \cup k_{2} B$ is not disjoint, we may say without loss of generality that $k_{2} b_{q}$ is in the numerical semigroup generated by the rest of the elements in $C$. That is, there are non negative integers $c_{i}$ for $1 \leq i \leq p$ and $d_{j}$ for $1 \leq j \leq q-1$ such that

$$
k_{2} b_{q}=\sum_{i=1}^{p} c_{i} k_{1} a_{i}+\sum_{j=1}^{q-1} d_{j} k_{2} b_{j} .
$$

Hence, $k_{2}$ divides $k_{1} \sum_{i=1}^{p} c_{i} a_{i}$ and, since $\left(k_{1}, k_{2}\right)=1$, one has that $\sum_{i=1}^{p} c_{i} a_{i}=k_{2} t$ for some non negative integer $t$. Moreover, $t$ must be positive or else $B$ will not be a minimal generating set for $\langle B\rangle$.

Dividing the above equation by $k_{2}$, we get

$$
b_{q}=k_{1} t+\sum_{j=1}^{q-1} d_{j} b_{j}
$$

Now, note that $k_{1}=\sum_{j=1}^{q} r_{j} b_{j}$ for some non negative integers $r_{j}$. Thus, we get

$$
\sum_{j=1}^{q-1}\left(t r_{j}+d_{j}\right) b_{j}+\left(t r_{q}-1\right) b_{q}=0 .
$$

Since $t r_{j}+d_{j} \geq 0$ and $t r_{q} \geq 0$, this can only occur if either $d_{j}=r_{j}=0$ for all $j=$ $1, \ldots, q-1$ and $t=r_{q}=1$, or $t r_{q}=0$. In the first case, one has that $b_{q}=k_{1} t=k_{1}$ which is impossible if $k_{1} \notin B$. On the other hand, $t r_{q}=0$ implies $r_{q}=0$. But then, $b_{q}=\sum_{j=1}^{q-1}\left(t r_{j}+d_{j}\right) b_{j}$ which contradicts the fact that $B$ is a minimal generating set for $\langle B\rangle$. Hence, $C=k_{1} A \sqcup k_{2} B$ minimally generates the semigroup $\langle C\rangle$ and we are done.

For a numerical semigroup, the definition that we gave in section 2.1 is equivalent to Rosales' original definition of gluing and this is proved in [16, Lemma 2.2]. We add our proof of the following result for completion and convenience:

Proposition 2.2 ([16, Lemma 2.2]). If $\langle C\rangle$ is obtained by gluing $\langle A\rangle$ and $\langle B\rangle$, then the ideal $I_{C}$ is minimally generated by the minimal generators of $I_{A}$ and $I_{B}$ and $\rho$, the binomial defined in section 2.2.

Proof. First note that $\sum_{i=1}^{p} c_{i}\left(k_{1} a_{i}\right)+\sum_{j=1}^{q} d_{j}\left(k_{2} b_{j}\right)=0$ for some integers $c_{i}, d_{j}$ is a minimal relation on the generators of the semigroup $\langle C\rangle$ if and only if $\prod_{c_{i}>0} x_{i}^{c_{i}} \prod_{d_{j}>0} y_{j}^{d_{j}}-$ $\prod_{c_{i}<0} x_{i}^{-c_{i}} \prod_{d_{j}<0} y_{j}^{-d_{j}}$ is a minimal generator for the ideal $I_{C}$.

With notations in section 2.2 , since

$$
\sum_{i=1}^{p} \alpha_{i}\left(k_{1} a_{i}\right)-\sum_{j=1}^{q} \beta_{j}\left(k_{2} b_{j}\right)=0
$$

for non negative integers $\alpha_{i}$ and $\beta_{j}$, one has that $\rho \in I_{C} \backslash\left(I_{A}+I_{B}\right)$. 
Let $\sigma=\sum_{i=1}^{p} c_{i}\left(k_{1} a_{i}\right)+\sum_{j=1}^{q} d_{j}\left(k_{2} b_{j}\right)=0$ be a relation on the elements in $C$ for some integers $c_{i}$ and $d_{j}$. Then, $k_{1}\left(\sum_{i=1}^{p} c_{i} a_{i}\right)=-k_{2}\left(\sum_{j=1}^{q} d_{j} b_{j}\right)$. Since $k_{1}$ and $k_{2}$ are relatively prime, we conclude that $\sum_{i=1}^{p} c_{i} a_{i}=k_{2} r=r \sum_{i=1}^{p} \alpha_{i} a_{i}$ and $\sum_{j=1}^{q} d_{j} b_{j}=-k_{1} r=$ $-r \sum_{j=1}^{q} \beta_{j} b_{j}$. So, $\sum_{i=1}^{p}\left(c_{i}-r \alpha_{i}\right) a_{i}=0=\sum_{j=1}^{q}\left(d_{j}+r \beta_{j}\right) b_{j}$. Hence, $\sigma=\sum_{i=1}^{p}\left(c_{i}-\right.$ $\left.r \alpha_{i}\right) k_{1} a_{i}+\sum_{j=1}^{q}\left(d_{j}+r \beta_{j}\right) k_{2} b_{j}$. Thus, any relation $\sigma$ among the integers in $C$ is a sum of relations among elements in $A$ and relations among elements $B$, unless $c_{i}=r \alpha_{i}$ and $d_{j}=-r b_{j}$ for all $i, j$. In that case, $\sigma$ is a multiple of the relation (11) and hence $I_{C} \subseteq$ $I_{A} R+I_{B} R+\langle\rho\rangle$. The other inclusion is trivial, and it is clear that taking minimal generators of $I_{A}$ and $I_{B}$ and adding $\rho$, one gets a sistem of generators of $I_{C}$ which is minimal.

\section{MAin RESUlt AND CONSEQUENCES}

With the notations in section [2.2, our main result is the following:

Theorem 3.1. Consider a numerical semigroup $\langle C\rangle$ minimally generated by $C$ and obtained by gluing $\langle A\rangle$ and $\langle B\rangle$ with $C=k_{1} A \sqcup k_{2} B$.

(1) $F_{A} \otimes F_{B}$ is a minimal graded free resolution of $R / I_{A} R+I_{B} R$.

(2) A minimal graded free resolution of the semigroup ring $k[C]$ can be obtained as the mapping cone of $\rho: F_{A} \otimes F_{B} \rightarrow F_{A} \otimes F_{B}$, where $\rho$ is induced by multiplication by $\rho$. In particular, $\left(I_{A} R+I_{B} R: \rho\right)=I_{A} R+I_{B} R$.

Proof. The minimal free resolutions $F_{A}$ and $F_{B}$ of $k[A] \simeq R_{A} / I_{A}$ and $k[B] \simeq R_{B} / I_{B}$ can be viewed as minimal free resolutions of the $R$-modules $R / I_{A} R$ and $R / I_{B} R$ (with the same maps). Since $I_{A} R$ and $I_{B} R$ are generated by elements involving disjoint sets of variables in $R, G=F_{A} \otimes F_{B}$ is a minimal graded free resolution of $R /\left(I_{A} R+I_{B} R\right)$.

Now consider the complex map on $G \rightarrow G$ induced by multiplication by $\rho$. All the maps in the complex are also multiplication by $\rho$ and hence this is a complex map. We note that $I_{A} R+I_{B} R+\rho$ is the prime ideal $I_{C}$ of height $n-1$. In the mapping cone $M(\rho)$ of the multiplication map $\rho: G \rightarrow G$, all of the fitting ideals contain $I_{A} R+I_{B} R+\rho$ up to radical and hence are of depth $n-1$. So, by the exactness criterion [5, Cor. 1], the mapping cone is acyclic and resolves $I_{C}=I_{A} R+I_{B} R+\rho$.

Minimality of our resolutions are straightforward. Since $F_{A}$ and $F_{B}$ are minimal, one has that $\partial_{i}^{A}\left(\left(F_{A}\right)_{i}\right) \subset\left(x_{1}, \ldots, x_{p}\right)\left(F_{A}\right)_{i-1}$ and $\partial_{j}^{B}\left(\left(F_{B}\right)_{j}\right) \subset\left(y_{1}, \ldots, y_{q}\right)\left(F_{B}\right)_{j-1}$ for $1 \leq i \leq$ $p-1$ and $1 \leq j \leq q-1$. Thus, denoting by $\partial_{\bullet}^{G}$ the differentials in $G$, one has that $\partial_{k}^{G}\left(G_{k}\right) \subset$ $\left(x_{1}, \ldots, x_{p}, y_{1}, \ldots y_{q}\right) G_{k-1}$ for all $k, 1 \leq k \leq p+q-2$, and hence $G$ resolves $R / I_{A} R+I_{B} R$ minimally. Finally, since the maps in the complex map $G \rightarrow G$ are all multiplication by $\rho$ and $\rho \in\left(x_{1}, \ldots, x_{p}, y_{1} \ldots, y_{q}\right)$, we see that if $\partial_{\bullet}$ are the differentials in the mapping cone $M(\rho)$, for all $k, 1 \leq k \leq p+q-1$, one has $\partial_{k}\left(M(\rho)_{k}\right) \subset\left(x_{1}, \ldots, x_{p}, y_{1}, \ldots y_{q}\right) M(\rho)_{k-1}$, and $M(\rho)$ resolves $R / I_{C}$ minimally.

As a consequence, we see that the $H_{1}(M(\rho))=0$ and hence $\left(I_{A} R+I_{B} R: \rho\right)=$ $I_{A} R+I_{B} R$ 
Example 1. Consider the decomposable numerical semigroup $\langle C\rangle$ generated by

$$
C=\{187,289,425,323,140,364,336\}=k_{1} A \sqcup k_{2} B
$$

with $A=\{11,17,25,19\}, B=\{5,13,12\}, k_{1}=17=5+12 \in\langle B\rangle$ and $k_{2}=28=$ $11+17 \in\langle A\rangle$. Using Singular [8], one gets that the ideal $I_{A} \subset R_{A}=k\left[x_{1}, \ldots, x_{4}\right]$ is minimally generated by 5 binomials, $f_{1}=x_{1} x_{3}-x_{2} x_{4}, f_{2}=x_{4}^{4}-x_{2}^{3} x_{3}, f_{3}=x_{2}^{4}-x_{1} x_{4}^{3}$, $f_{4}=x_{3}^{2}-x_{1}^{3} x_{2}, f_{5}=x_{1}^{4}-x_{3} x_{4}$, the ring $k[A] \simeq R_{A} / I_{A}$ is Gorenstein, and its minimal free resolution is

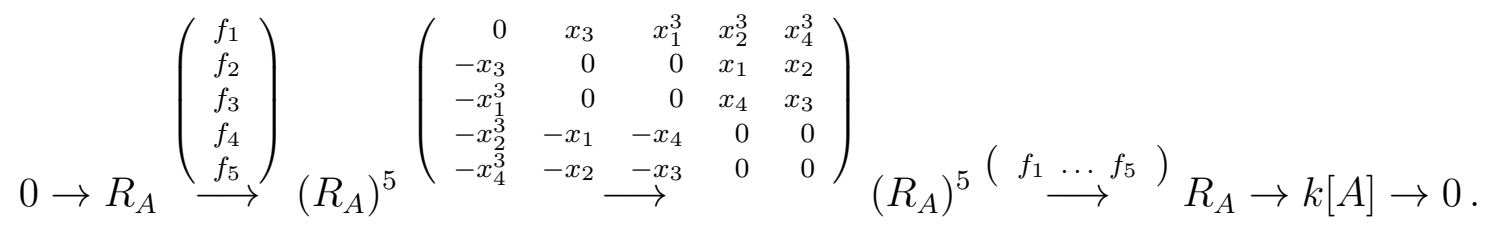

The ideal $I_{B} \subset R_{B}=k\left[y_{1}, y_{2}, y_{3}\right]$ is generated by 3 binomials, $g_{1}=y_{3}^{3}-y_{1}^{2} y_{2}^{2}, g_{2}=y_{1}^{5}-y_{2} y_{3}$, $g_{3}=y_{2}^{3}-y_{1}^{3} y_{3}^{2}$, it is Hilbert-Burch, and its minimal free resolution is

$$
\left.0 \rightarrow\left(R_{B}\right)^{2} \stackrel{\left(\begin{array}{ll}
y_{1}^{3} & y_{2} \\
y_{2}^{2} & y_{3}^{2} \\
y_{3} & y_{1}^{2}
\end{array}\right)}{\longrightarrow}\left(R_{B}\right)^{3} \stackrel{\left(g_{1} g_{2} g_{3}\right.}{\longrightarrow}\right) R_{B} \rightarrow k[B] \rightarrow 0 .
$$

The tensor product of these two resolutions provides a minimal free resolution of $R / J$ where $J=I_{A} R+I_{B} R$ :

$$
0 \rightarrow R^{2} \rightarrow R^{13} \rightarrow R^{26} \rightarrow R^{22} \rightarrow R^{8} \rightarrow R \rightarrow R / J \rightarrow 0
$$

Note that the differentials can be easily written down if needed. Finally, the extra minimal generator in $I_{C}$ is $\rho=x_{1} x_{2}-y_{1} y_{3}$ and the mapping cone induced by multiplication by $\rho$ gives a minimal resolution of $k[C]$,

$$
0 \rightarrow R^{2} \rightarrow R^{15} \rightarrow R^{39} \rightarrow R^{48} \rightarrow R^{30} \rightarrow R^{9} \rightarrow R \rightarrow k[C] \rightarrow 0 .
$$

Again, the differentials are known.

Of course, the differentials in the resolution of $k[C]$ depend on $k_{1}$ and $k_{2}$ when the numerical semigroup decomposes as $C=k_{1} A \sqcup k_{2} B$ but the Betti numbers do not as our next result shows. For any $R$-module $M$, we denote by $\beta_{i}(M)$ its Betti numbers with the convention that $\beta_{i}(M)=0$ for $i<0$ and $i>\operatorname{pd}(M)$, the projective dimension of $M$. For the semigroup ring $k[C]$ associated to a numerical semigroup $\langle C\rangle$, we will use the notation $\beta_{i}(C)=\beta_{i}(k[C])$.

Corollary 3.2. If the numerical semigroup $\langle C\rangle$ is obtained by gluing $\langle A\rangle$ and $\langle B\rangle$, then

$$
\forall i \geq 0, \beta_{i}(C)=\sum_{i^{\prime}=0}^{i} \beta_{i^{\prime}}(A)\left[\beta_{i-i^{\prime}}(B)+\beta_{i-i^{\prime}-1}(B)\right]
$$

Proof. Set $J=I_{A} R+I_{B} R$. Since the resolution of $R / J$ is the tensor product of the resolutions of $k[A]$ and $k[B], \beta_{i}(R / J)=\sum_{i^{\prime}=0}^{i} \beta_{i^{\prime}}(A) \beta_{i-i^{\prime}}(B)$ for $i=0, \ldots, p+q-2$. One 
can write $\beta_{i}(R / J)=\sum_{i^{\prime}>0} \beta_{i^{\prime}}(A) \beta_{i-i^{\prime}}(B)$ because $\beta_{i-i^{\prime}}(B)=0$ if $i^{\prime}>i$, and this formula holds for all $i \geq 0$ since for $i>p+q-2$, either $i^{\prime}>p-1$ or $i-i^{\prime}>q-1$ and hence $\beta_{i^{\prime}}(A) \beta_{i-i^{\prime}}(B)=0$. Note in particular that $\beta_{p+q-2}(R / J)=\beta_{p-1}(A) \beta_{q-1}(B)$. Now the resolution of $k[C]$ is the mapping cone of the resolution of $R / J$ induced by multiplication by $\rho$ and hence $\beta_{0}(C)=1, \beta_{i}(C)=\beta_{i}(R / J)+\beta_{i-1}(R / J)$ for $i=1, \ldots, p+q-2$, and $\beta_{p+q-1}(C)=\beta_{p+q-2}(R / J)$, and the result follows.

Remark 1. In Corollary [3.2, $A$ and $B$ play the same role and hence the symmetric formula for the Betti numbers also holds:

$$
\forall i \geq 0, \beta_{i}(C)=\sum_{i^{\prime}=0}^{i} \beta_{i^{\prime}}(B)\left[\beta_{i-i^{\prime}}(A)+\beta_{i-i^{\prime}-1}(A)\right] .
$$

Example 2. Consider the decomposable numerical semigroup $\langle C\rangle$ generated by

$$
C=\{450,522,612,576,305,793,732\}=k_{1} A \sqcup k_{2} B
$$

with $A=\{25,29,34,32\}, B=\{5,13,12\}, k_{1}=18=5+13 \in\langle B\rangle$ and $k_{2}=61=$ $29+32 \in\langle A\rangle$. Using Singular, one gets that the Betti numbers of $k[A]$ and $k[B]$ are

\begin{tabular}{|c|c|c|c|c|}
\hline$i$ & 0 & 1 & 2 & 3 \\
\hline$\beta_{i}(A)$ & 1 & 7 & 10 & 4 \\
\hline
\end{tabular}

\begin{tabular}{|c|l|l|l|}
\hline$i$ & 0 & 1 & 2 \\
\hline$\beta_{i}(B)$ & 1 & 3 & 2 \\
\hline
\end{tabular}

Applying the formula in Corollary 3.2 and the remark after the corollary, one gets that $\beta_{0}(C)=1$, and

$$
\begin{aligned}
& \beta_{1}(C)=1(3+1)+7.1=1(7+1)+3.1=11, \\
& \beta_{2}(C)=1(2+3)+7(3+1)+10.1=1 .(10+7)+3(7+1)+2.1=43, \\
& \beta_{3}(C)=1.2+7(2+3)+10(3+1)+4.1=1 .(4+10)+3(10+7)+2(7+1)=81, \\
& \beta_{4}(C)=7.2+10(2+3)+4(3+1)=1.4+3(4+10)+2(10+7)=80, \\
& \beta_{5}(C)=10.2+4(2+3)=3.4+2(4+10)=40, \\
& \beta_{6}(C)=4.2=8,
\end{aligned}
$$

and the minimal free resolution of $k[C]$ shows as

$$
0 \rightarrow R^{8} \rightarrow R^{40} \rightarrow R^{80} \rightarrow R^{81} \rightarrow R^{43} \rightarrow R^{11} \rightarrow R \rightarrow k[C] \rightarrow 0 .
$$

As a direct consequence of Corollary 3.2 , one gets that if $A$ and $B$ have $p$ and $q$ elements respectively, then the last nonzero Betti number of $k[C]$ is $\beta_{p+q-1}(C)=\beta_{p-1}(A) \beta_{q-1}(B)$. In other words, one has the following result:

Corollary 3.3. If the numerical semigroup $\langle C\rangle$ is obtained by gluing two numerical semigroups $\langle A\rangle$ and $\langle B\rangle$, then the Cohen-Macaulay type of $k[C]$ is the product of the CohenMacaulay types of $k[A]$ and $k[B]$.

The type of a numerical semigroup $\langle C\rangle$ is defined in [11] as the number of elements in the set $C^{\prime}=\{x \in \mathbb{Z} / x \notin\langle C\rangle$ and $x+s \in\langle C\rangle, \forall s \in\langle C\rangle \backslash\{0\}\}$. In [15. Prop. 6.6] it is shown that the type of a numerical semigroup obtained by gluing two semigroups is the product of the types of the two semigroups. In order to see that this is the same result 
as Corollary 3.3 , we need to show the following result that we will prove since we could not find any reference for it.

Lemma 3.4. The type of any numerical semigroup $\langle C\rangle$ coincides with the Cohen-Macaulay type of its semigroup ring $k[C]$.

Proof. The Cohen-Macaulay type of any simplicial Cohen-Macaulay semigroup is computed in [7, Thm. 4.2(ii)]. Applying this result to a numerical semigroup $\langle C\rangle$ minimally generated by $C$, if $e$ is the smallest element in $C$ and $A=C \backslash\{e\}$, the Cohen-Macaulay type of $k[C]$ is the number of elements in the set $Q=\{q \in\langle C\rangle / q-e \notin\langle C\rangle\}$ that are maximal in the sence that $q+a \notin Q$ for all $a \in A$. Now one can easily check that $q \in Q$ is maximal if and only if $q-e \in S^{\prime}$, and the result follows.

The following corollary gives more direct consequences of Corollaries 3.2 and 3.3 ;

Corollary 3.5. Let $\langle C\rangle$ be a numerical semigroup obtained by gluing $\langle A\rangle$ and $\langle B\rangle$.

(1) $k[C]$ is Gorenstein, respectively a complete intersection, if and only if $k[A]$ and $k[B]$ are both Gorenstein, respectively complete intersections.

(2) If neither $k[A]$ nor $k[B]$ is Gorenstein, then the Cohen-Macaulay type of $k[C]$ is not prime.

Remark 2. The result for complete intersections is stronger than what is stated in Corollary 3.5 (11): every complete intersection numerical semigroup is indeed the gluing of two complete intersection subsemigroups. This was shown in [16] and previously in [9]; see [17, Thm. 9.10]. Note that this result does not hold for Gorenstein semigroups since there exist indecomposable Gorenstein semigroups when the embedding dimension is $\geq 4$ as we will see later in example 6.

One can be more precise and compute the graded Betti numbers of a decomposable numerical semigroup. For any graded $R$-module $M$, we denote by $\beta_{i j}(M)$ the number of syzygies of degree $j$ at the $i$-th step of a minimal graded free resolution. The set of integers $\left\{\beta_{i j}(M)\right\}$ are the graded Betti numbers of $M$. As for global Betti numbers, for the semigroup ring $k[C]$ associated to a numerical semigroup $\langle C\rangle$, we will use the notation $\beta_{i j}(C)=\beta_{i j}(k[C])$.

Proposition 3.6. If the numerical semigroup $\langle C\rangle$ decomposes as $C=k_{1} A \sqcup k_{2} B$, then

$$
\beta_{i, j}(C)=\sum_{i^{\prime}=0}^{i}\left(\sum_{r, s / k_{1} r+k_{2} s=j} \beta_{i^{\prime} r}(A)\left[\beta_{i-i^{\prime}, s}(B)+\beta_{i-i^{\prime}-1, s-k_{1}}(B)\right]\right) .
$$

Proof. The graded Betti numbers of $\left\langle k_{1} A\right\rangle$ and $\left\langle k_{2} B\right\rangle$ are $\beta_{i, k_{1} j}\left(k_{1} A\right)=\beta_{i j}(A)$ and $\beta_{i, k_{2} j}\left(k_{2} B\right)=\beta_{i j}(B)$. Set $J=I_{A} R+I_{B} R$. The resolution of $R / J$ is $F_{A} \otimes F_{B}$ and hence, at the $i$-th step of the resolution, for $1 \leq i \leq p+q-2$, there will be a generator of degree $k_{1} r+k_{2} s$ any time $\beta_{i^{\prime}, r}(A) \neq 0$ and $\beta_{i-i^{\prime}, s}(B) \neq 0$ for some $i^{\prime}, 0 \leq i^{\prime} \leq i$. In other words, if we set $t_{i}(A)=\left\{j \in \mathbb{N} / \beta_{i j}(A) \neq 0\right\}$ for all $i \in\{0, \ldots, p-1\}$ and 
$t_{i}(B)=\left\{j \in \mathbb{N} / \beta_{i j}(B) \neq 0\right\}$ for all $i \in\{0, \ldots, q-1\}$, the minimal free resolution of $R / J$ is

$$
0 \rightarrow F_{p+q-2} \rightarrow \cdots F_{i} \rightarrow F_{i-1} \rightarrow \cdots \rightarrow F_{1} \rightarrow R \rightarrow R / J \rightarrow 0
$$

with $F_{i}=\bigoplus_{i^{\prime}=0}^{i}\left(\bigoplus_{\substack{r \in t_{i^{\prime}}(A) \\ s \in t_{i-i^{\prime}}(B)}} R\left(-k_{1} r-k_{2} s\right)^{\beta_{i^{\prime} r}(A) \beta_{i-i^{\prime}, s}(B)}\right)$. Thus, the graded Betti numbers of $R / J$ are, for $0 \leq i \leq p+q-2$,

$$
\beta_{i, j}(R / J)=\sum_{i^{\prime}=0}^{i}\left(\sum_{\substack{r \in t_{i^{\prime}}(A), s \in t_{i-i^{\prime}}(B) / \\ j=k_{1} r+k_{2} s}} \beta_{i^{\prime} r}(A) \beta_{i-i^{\prime}, s}(B)\right) .
$$

Now the resolution of $k[C]$ is the mapping cone of $\rho: F_{A} \otimes F_{B} \rightarrow F_{A} \otimes F_{B}$ induced by multiplication by $\rho$ which has degree $k_{1} k_{2}$. Hence the graded Betti numbers of $k[C]$ are $\beta_{00}(C)=1, \beta_{i j}(C)=\beta_{i j}(R / J)+\beta_{i-1, j-k_{1} k_{2}}(R / J)$ for $1 \leq i \leq p+q-2$, and $\beta_{p+q-1, j}(C)=\beta_{p+q-2, j-k_{1} k_{2}}(R / J)$, and we have, for all $i, j \geq 0$,

$$
\begin{aligned}
\beta_{i j}(C) & =\sum_{i^{\prime}=0}^{i}\left(\sum_{r, s / j=k_{1} r+k_{2} s} \beta_{i^{\prime} r}(A) \beta_{i-i^{\prime}, s}(B)\right)+\sum_{i^{\prime}=0}^{i-1}\left(\sum_{r, s / j=k_{1} r+k_{2} s+k_{1} k_{2}} \beta_{i^{\prime} r}(A) \beta_{i-i^{\prime}-1, s}(B)\right) \\
& =\sum_{i^{\prime}=0}^{i}\left(\sum_{r, s / j=k_{1} r+k_{2} s} \beta_{i^{\prime} r}(A) \beta_{i-i^{\prime}, s}(B)\right)+\sum_{i^{\prime}=0}^{i}\left(\sum_{r, s / j=k_{1} r+k_{2} s} \beta_{i^{\prime} r}(A) \beta_{i-i^{\prime}-1, s-k_{1}}(B)\right)
\end{aligned}
$$

because in the second summand, for $i^{\prime}=i$, one has $i-i^{\prime}-1<0$, and the result follows.

Remark 3. As for Corollary [3.2, the formula obtained by exchanging $A$ and $B$ in the formula in Proposition 3.6 also holds.

For any graded $R$-module $M$, the Castelnuovo-Mumford regularity (or regularity for short) of $M, \operatorname{reg}(M)$, is

$$
\operatorname{reg}(M)=\max \left\{j-i, i \geq 0, j \geq 0 / \beta_{i j}(M) \neq 0\right\} .
$$

Again, for the semigroup ring $k[C]$ associated to a numerical semigroup $\langle C\rangle$, we will use the notation $\operatorname{reg}(C)=\operatorname{reg}(k[C])$. In this context, the regularity is sometimes called the weighted regularity since we give weights to the variables to make our semigroup rings graded modules over the corresponding polynomial ring.

Corollary 3.7. If the numerical semigroup $\langle C\rangle$ is the gluing of two numerical semigroups $\langle A\rangle$ and $\langle B\rangle$ with $C=k_{1} A \sqcup k_{2} B, \# A=p$ and $\# B=q$, then

$$
\operatorname{reg}(C)=k_{1} \operatorname{reg}(A)+k_{2} \operatorname{reg}(B)+(p-1)\left(k_{1}-1\right)+(q-1)\left(k_{2}-1\right)+k_{1} k_{2}-1 .
$$

Proof. Since $k[A], k[B]$ and $k[C]$ are Cohen-Macaulay, their regularity is attained at the last step of their minimal graded free resolutions, i.e. $\operatorname{reg}(A)=\delta_{A}-(p-1), \operatorname{reg}(B)=$ $\delta_{B}-(q-1)$, and $\operatorname{reg}(C)=\delta_{C}-(p+q-1)$ where $\delta_{A}, \delta_{B}$ and $\delta_{C}$ is the maximal degree of a syzygy in the last step of a minimal graded free resolution of $k[A], k[B]$ and $k[C]$ 
respectively. According to the argument in the proof of Proposition 3.6, the maximal degree of a syzygy in the last step of a minimal graded free resolution of $R / I_{A} R+I_{B} R$ is $k_{1} \delta_{A}+k_{2} \delta_{B}$, and hence $\delta_{C}=k_{1} \delta_{A}+k_{2} \delta_{B}+k_{1} k_{2}$, and the result follows.

Example 3. Computing the degrees of all the syzygies in the resolutions of $k[A]$ and $k[B]$ in example 1, one gets that $\operatorname{reg}(A)=134$ and $\operatorname{reg}(B)=49$. The formula in Corollary 3.7 then gives that $\operatorname{reg}(C)=4227$ which can be checked using Singular.

For a numerical semigroup $\langle C\rangle$, denote by $H_{C}(t)$ the Hilbert series of the ring $k[C]$. Using the well-known relation between graded Betti numbers and Hilbert series (see, e.g., [10, Thm. 1.11]), we recover the formula in [1, Corollary 16].

Corollary 3.8. If the numerical semigroup $\langle C\rangle$ is the gluing of two numerical semigroups $\langle A\rangle$ and $\langle B\rangle$ with $C=k_{1} A \sqcup k_{2} B$, then $H_{C}(t)=\left(1-t^{k_{1} k_{2}}\right) H_{A}\left(t^{k_{1}}\right) H_{B}\left(t^{k_{2}}\right)$.

Proof. Set $J=I_{A} R+I_{B} R$. The Hilbert series of $R / J$ is

$$
H_{R / J}(t)=\frac{\sum_{j}\left(\sum_{i=0}^{p+q-2}(-1)^{i} \beta_{i j}(R / J) t^{j}\right)}{\prod_{i=1}^{p}\left(1-t^{k_{1} a_{i}}\right) \prod_{i=1}^{q}\left(1-t^{k_{2} b_{i}}\right)} .
$$

The numerator of this series, denoted by $N_{R / J}(t)$, can be computed using the formula for the graded Betti numbers of $R / J$ obtained in the proof of Proposition 3.6. Using the sets $t_{i}(A)$ and $t_{i}(B)$ defined there, one has

$$
\begin{aligned}
N_{R / J}(t) & =\sum_{i=0}^{p+q-2}(-1)^{i}\left(\sum_{i^{\prime}=0}^{i} \sum_{\substack{r \in t_{i^{\prime}}(A) \\
s \in t_{i-i^{\prime}}(B)}} \beta_{i^{\prime} r}(A) \beta_{i-i^{\prime}, s}(B) t^{k_{1} r+k_{2} s}\right) \\
& =\sum_{i=0}^{p+q-2} \sum_{i^{\prime}=0}^{i}\left(\sum_{\substack{r \in t_{i^{\prime}}(A) \\
s \in t_{i-i^{\prime}}(B)}}(-1)^{i^{\prime}} \beta_{i^{\prime}, r}(A) t^{k_{1} r} \times(-1)^{i-i^{\prime}} \beta_{i-i^{\prime}, s}(B) t^{k_{2} s}\right) \\
& =\left(\sum_{i=0}^{p-1}(-1)^{i} \sum_{r \in t_{i}(A)} \beta_{i r}(A) t^{k_{1} r}\right)\left(\sum_{i=0}^{q-1}(-1)^{i} \sum_{s \in t_{i}(B)} \beta_{i s}(B) t^{k_{2} s}\right)
\end{aligned}
$$

and hence $H_{R / J}(t)=H_{A}\left(k_{1} t\right) \times H_{B}\left(k_{2} t\right)$. On the other hand, the Hilbert Series of $k[C]$ is

$$
H_{C}(t)=\frac{\sum_{j}\left(\sum_{i=0}^{p+q-1}(-1)^{i} \beta_{i j}(C) t^{j}\right)}{\prod_{i=1}^{p}\left(1-t^{k_{1} a_{i}}\right) \prod_{i=1}^{q}\left(1-t^{k_{2} b_{i}}\right)}
$$


and, since for all $i, j \geq 0, \beta_{i j}(C)=\beta_{i j}(R / J)+\beta_{i-1, j-k_{1} k_{2}}(R / J)$, the numerator of this series, $N_{C}(t)$, is

$$
\begin{aligned}
N_{C}(t) & =\sum_{j}\left(\sum_{i=0}^{p+q-2}(-1)^{i} \beta_{i j}(R / J) t^{j}\right)+\sum_{j}\left(\sum_{i=1}^{p+q-1}(-1)^{i} \beta_{i-1, j-k_{1} k_{2}}(R / J) t^{j}\right) \\
& =\sum_{j}\left(\sum_{i=0}^{p+q-2}(-1)^{i} \beta_{i j}(R / J) t^{j}\right)+\sum_{j}\left(\sum_{i=0}^{p+q-2}(-1)^{i+1} \beta_{i j}(R / J) t^{j+k_{1} k_{2}}\right) \\
& =\left(1-t^{k_{1} k_{2}}\right) N_{R / J}(t),
\end{aligned}
$$

and hence $H_{C}(t)=\left(1-t^{k_{1} k_{2}}\right) H_{R / J}(t)$, and the result follows.

One can also show that the minimal free resolution of $k[C]$ constructed in Theorem 3.1 inherits the DG algebra structure of $k[A]$ and $k[B]$.

A complex of free $R$-modules, $\mathbf{F}: \ldots \rightarrow F_{i} \stackrel{d_{i}}{\rightarrow} F_{i-1} \ldots \rightarrow F_{1} \rightarrow F_{0}=R$ is said to have a differential graded algebra structure ( $D G$ algebra structure for short) if there is a multiplication $*$ on $F=\oplus F_{i}$ which makes it an associative and graded commutative differential graded algebra; see [6]. This means that $*$ is associative and, for all $a \in F_{i}$ and $b \in F_{j}, a * b \in F_{i+j}, b * a=(-1)^{i+j} a * b$, and $d_{i+j}(a * b)=d_{i}(a) * b+(-1)^{i} a * d_{j}(b)$. This last equality is called the Leibnitz rule. When a resolution of an $R$-algebra has a DG algebra structure, we say that it is a $D G$ algebra resolution. For a detailed study of these multiplicative structures on resolutions, see, e.g., [2].

In our case, using the construction in Theorem 3.1, we can write the multiplicative structure explicitly.

Proposition 3.9. Suppose that the numerical semigroup $\langle C\rangle$ is obtained by gluing $\langle A\rangle$ and $\langle B\rangle$. If the semigroup rings $k[A]$ and $k[B]$ have minimal $D G$ algebra resolutions, then so does the semigroup ring $k[C]$.

Proof. Assume that $F_{A}$ and $F_{B}$ in section 2.2 have a DG algebra structure over $R_{A}$ and $R_{B}$ respectively. Then $F_{A} \otimes F_{B}$, which is a resolution of $R / I_{A} R+I_{B} R$, admits the following DG algebra structure: let $a_{1} \otimes b_{1} \in\left(F_{A}\right)_{i} \otimes\left(F_{B}\right)_{r-i}$ and $a_{2} \otimes b_{2} \in\left(F_{A}\right)_{j} \otimes\left(F_{B}\right)_{s-j}$ be two basis elements in $\left(F_{A} \otimes F_{B}\right)_{r}$ and $\left(F_{A} \otimes F_{B}\right)_{s}$ respectively, and consider the following product:

$$
\left(a_{1} \otimes b_{1}\right)\left(a_{2} \otimes b_{2}\right)=(-1)^{(r-i) j}\left(a_{1} a_{2}\right) \otimes\left(b_{1} b_{2}\right)
$$

One has that

$$
\begin{aligned}
\left(a_{2} \otimes b_{2}\right)\left(a_{1} \otimes b_{1}\right) & =(-1)^{(s-j) i+i j+(r-i)(s-j)}\left(a_{1} a_{2}\right) \otimes\left(b_{1} b_{2}\right) \\
& =(-1)^{r s+(r-i) j} a_{1} a_{2} \otimes b_{1} b_{2}=(-1)^{r s}\left(a_{1} \otimes b_{1}\right)\left(a_{2} \otimes b_{2}\right) .
\end{aligned}
$$


Further, for $w=\left(a_{1} \otimes b_{1}\right)\left(a_{2} \otimes b_{2}\right)$, if $\partial$ denotes the differential, one has that

$$
\begin{aligned}
& \partial w=(-1)^{(r-i) j}\left[\left(\partial a_{1} a_{2}+(-1)^{i} a_{1} \partial a_{2}\right) \otimes b_{1} b_{2}+\right. \\
&\left.(-1)^{i+j} a_{1} a_{2} \otimes\left(\partial b_{1} b_{2}+(-1)^{r-i} b_{1} \partial b_{2}\right)\right] \\
&=\left(\partial a_{1} \otimes b_{1}\right)\left(a_{2} \otimes b_{2}\right)+(-1)^{i}\left(a_{1} \otimes \partial b_{1}\right)\left(a_{2} \otimes b_{2}\right)+ \\
&(-1)^{r}\left(a_{1} \otimes b_{1}\right)\left(\partial a_{2} \otimes b_{2}\right)+(-1)^{r+j}\left(a_{1} \otimes b_{1}\right)\left(a_{2} \otimes \partial b_{2}\right) \\
&=\left(\partial\left(a_{1} \otimes b_{1}\right)\right)\left(a_{2} \otimes b_{2}\right)+(-1)^{r}\left(a_{1} \otimes b_{1}\right)\left(\partial\left(a_{2} \otimes b_{2}\right)\right)
\end{aligned}
$$

and hence it satisfies the Leibnitz rule. To check associativity, all we need to check is the sign. As the equality $\operatorname{deg} b_{1} \operatorname{deg} a_{2}+\left(\operatorname{deg} b_{1}+\operatorname{deg} b_{2}\right) \operatorname{deg} a_{3}=\operatorname{deg} b_{1}\left(\operatorname{deg} a_{2}+\operatorname{deg} a_{3}\right)+$ $\operatorname{deg} b_{2} \operatorname{deg} a_{3}$ always holds, we are done and hence $F_{A} \otimes F_{B}$ is a DG algebra resolution of $R / I_{A} R+I_{B} R$ with this product.

Denote the complex $F_{A} \otimes F_{B}$ by $G$ for convenience. We have a complex map $\rho: G \rightarrow G$ given by multiplication by $\rho$. Then the mapping cone of $\rho$ has a multiplicative structure $\star: G_{i} \oplus G_{i-1} \times G_{j} \oplus G_{j-1} \rightarrow G_{i+j} \oplus G_{i+j-1}$ given by

$$
\left(a_{1}, b_{1}\right) \star\left(a_{2}, b_{2}\right)=\left(a_{1} a_{2}, a_{1} b_{2}+(-1)^{j} b_{1} a_{2}\right) .
$$

For the sake of completion, we verify that this provides a multiplicative structure.

$$
\begin{aligned}
\left(a_{2}, b_{2}\right) \star\left(a_{1}, b_{1}\right) & =\left(a_{2} a_{1}, a_{2} b_{1}+(-1)^{i} b_{2} a_{1}\right)=(-1)^{i j}\left(a_{1} a_{2}, a_{1} b_{2}+(-1)^{j} b_{1} a_{2}\right) \\
& =(-1)^{i j}\left(a_{1}, b_{1}\right) \star\left(a_{2}, b_{2}\right)
\end{aligned}
$$

so it is graded commutative. It is associative because $\left(a_{1}, b_{1}\right) \star\left(a_{2}, b_{2}\right) \star\left(a_{3}, b_{3}\right)=$ $\left(a_{1} a_{2} a_{3}, a_{1} a_{2} b_{3}+(-1)^{k} a_{1} b_{2} a_{3}+(-1)^{(j+k)} b_{1} a_{2} a_{3}\right)$.

Finally, to check the Leibnitz rule, we see that for $w=\left(a_{1}, b_{1}\right) \star\left(a_{2}, b_{2}\right)$, denoting again the differential by $\partial$, we have

$$
\begin{aligned}
\partial w= & \left(\partial\left(a_{1} a_{2}\right)+(-1)^{i+j-1} \rho\left(a_{1} b_{2}+(-1)^{j} b_{1} a_{2}\right), \partial\left(a_{1} b_{2}+(-1)^{j} b_{1} a_{2}\right)\right) \\
= & \left(\partial\left(a_{1}\right) a_{2}+(-1)^{i} a_{1} \partial\left(a_{2}\right)+(-1)^{i+j-1} \rho\left(a_{1} b_{2}+(-1)^{j} b_{1} a_{2}\right),\right. \\
& \left.\partial\left(a_{1}\right) b_{2}+(-1)^{i} a_{1} \partial\left(b_{2}\right)+(-1)^{j}\left(\partial\left(b_{1}\right) a_{2}+(-1)^{i-1} b_{1} \partial\left(a_{2}\right)\right)\right) \\
= & \left(\partial\left(a_{1}\right)+(-1)^{i-1} \rho b_{1}, \partial\left(b_{1}\right)\right) \star\left(a_{2}, b_{2}\right)+ \\
& (-1)^{i}\left(a_{1}, b_{1}\right) \star\left(\partial\left(a_{2}\right)+(-1)^{j-1} \rho b_{2}, \partial\left(b_{2}\right)\right) .
\end{aligned}
$$

This completes the proof.

Remark 4. In fact, the following general result is true: if $C_{1}$ and $C_{2}$ are two complexes with a DG algebra structure, if $C_{2}$ has a structure of $C_{1}$-module, and if $\phi: C_{1} \rightarrow C_{2}$ is a complex map which is also a $C_{2}$-module homomorphism, then the mapping cone of $\phi$ has a structure of DG algebra. This can be applied at the end of the proof of Theorem 3.9 to $C_{1}=C_{2}=F_{A} \otimes F_{B}$ since the map $\rho$ is clearly a module homomorphism because it is the multiplication by an element of the ring $R$. We gave a direct proof of the specific result above for completion. 


\section{Simple SPLiT}

Let's focus here on a special class of decomposable numerical semigroups: if the numerical semigroup $\langle C\rangle$ decomposes as $C=k_{1}\{1\} \sqcup k_{2} A$ where $k_{1} \in\langle A\rangle \backslash A, k_{2} \neq 1$ and $\operatorname{gcd}\left(k_{1}, k_{2}\right)=1$, we call the decomposition a simple split and denote $C=k_{1} \sqcup k_{2} A$ for simplicity. The structure of the resolution of $k[C]$ and the value of its Betti numbers come as a direct consequence of Theorem 3.1 and Corollary 3.2, but it can be obtained, in this particular case, by a simple and direct argument.

Theorem 4.1. Suppose that $C=k_{1} \sqcup k_{2} A$ is a simple split. Then, a minimal free resolution of the semigroup ring $k[C]$ is obtained as the mapping cone over a minimal resolution of $k[A]$ induced by multiplication by a single element. In particular, $\beta_{i}(C)=$ $\beta_{i}(A)+\beta_{i-1}(A)$, and the Cohen-Macaulay type of $k[C]$ is the same as the type of $k[A]$.

Proof. Assume that the embedding dimension of $A$ is $n, A=\left\{a_{1}, \ldots, a_{n}\right\}$, and denote $R=k\left[x_{0}, \ldots, x_{n}\right]$. The following diagram commutes:

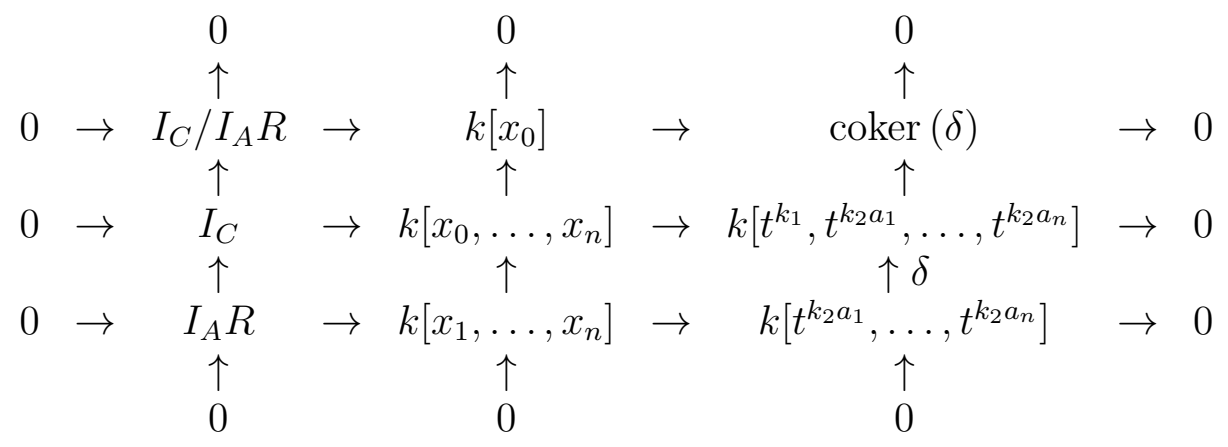

The first row of the diagram makes $I_{C} / I_{A} R$ an ideal of $k\left[x_{0}\right]$ which is hence principal: it is generated by the class of the element $\rho=x_{0}^{k_{1}}-x_{1}^{\alpha_{1}} \ldots x_{n}^{\alpha_{n}}$ coming from the fact that $k_{1} \in\langle A\rangle$ and so $k_{1}=\alpha_{1} a_{1}+\cdots+\alpha_{n} a_{n}$. Let $F_{A}: 0 \rightarrow F_{n} \stackrel{\partial_{n}}{\rightarrow} F_{n-1} \rightarrow \ldots F_{i} \stackrel{\partial_{i}}{\rightarrow}$ $F_{i-1} \rightarrow \ldots F_{1} \rightarrow R$ be a minimal resolution of $R / I_{A} R$ and consider the complex map $\rho: F_{A} \rightarrow F_{A}$ given by multiplication by $\rho$. Now, $R / I_{A} R$ is Cohen Macaulay so all the maps $\partial_{i}: F_{i} \rightarrow F_{i-1}$ in the resolution have Fitting ideals equal up to radical to $I_{A}$ and hence have the same depth $n$. This follows from [18, Cor. 2.5]. The $i$ th Fitting ideal of the mapping cone is $I\left(\partial_{i}\right) I\left(\partial_{i-1}\right)+(\rho)$ and has depth $n+1$ since $\left.\rho \notin \sqrt{(} I_{A}\right)$. So, the mapping cone of the complex map $\rho$ is exact and it resolves $R / I_{A} R+(\rho)=R / I_{C}$.

These simple splits will appear in the next section when we describe decomposable numerical semigroups with small embedding dimension. One can also use simple splits to construct easily complete intersections of higher embedding dimension by adding generators in the semigroup one by one as the following example shows.

Example 4. The semigroup $\langle A\rangle$ minimally generated by the simple split $A=\{11\} \sqcup$ $2\{3,8\}=\{11,6,16\}$ is a complete intersection of embedding dimension 3 . One gets a complete intersection semigroup $\langle C\rangle$ of embedding dimension 4 by considering the following simple split $C=17 \sqcup 2 A=\{17,22,12,32\}$. One can iterate the procedure by 
considering, for example, the simple split $29 \sqcup 2 C$ to get a complete intersection of embedding dimension 5 .

Example 5. Complete intersections with embedding dimension 4 can be simple splits or not. The decomposable semigroup $\langle C\rangle$ with $C=11\{3,5\} \sqcup 8\{4,7\}=\{33,55,32,56\}$ is a complete intersection since the ideal $I_{C}$ is generated by the binomial $x_{1}^{5}-x_{2}^{3}$ that generates the ideal $I_{\langle 3,5\rangle}$, the binomial $x_{3}^{7}-x_{4}^{4}$ that generates the ideal $I_{\langle 4,7\rangle}$, and the binomial $\rho=x_{1} x_{2}-x_{3} x_{4}$ but it is not a simple split. The resolution of $k[C] \simeq R / I_{C}$, as the one of $k[C]$ in example 4, is a Koszul complex and it has the following form: $0 \rightarrow R \rightarrow R^{3} \rightarrow R^{3} \rightarrow R \rightarrow k[C] \rightarrow 0$.

\section{Semigroups With small Embedding Dimension}

Let $\langle C\rangle$ be a numerical semigroup minimally generated by $C$. Then the embedding dimension of the semigroup ring $\langle C\rangle$ is $n$. When $n=2,\langle C\rangle$ is a complete intersection, and when $n=3$, then $\langle C\rangle$ is either indecomposable or a complete intersection by [14]. In fact, when it is indecomposable of embedding dimension 3 , it is generated by the $2 \times 2$ minors of a $2 \times 3$ matrix of the form given in the introduction, just like the semigroup $\langle B\rangle$ in our examples 1 and 2, This matrix is also the transpose of the matrix of the first syzygies of the ideal $I_{C}$. Such ideals are called Hilbert-Burch since they fit in the class of ideals satisfying the theorem of the same name; see [10, Thm. 3.2].

For embedding dimension 4 we have the following:

Proposition 5.1. Numerical semigroups of embedding dimension 4 that are decomposable are either complete intersections or almost complete intersections of type 2.

Proof. When $\langle C\rangle$ is decomposable of embedding dimension 4, if $\langle C\rangle$ is not a simple split then it decomposes as $C=k_{1} A \sqcup k_{2} B$ where both $A$ and $B$ have 2 elements (as in example 5) and $\langle C\rangle$ is a complete intersection as the gluing of two complete intersections. If $\langle C\rangle$ is a simple split, $k_{1} \sqcup k_{2} A$, and $\langle A\rangle$ is a complete intersection (as in example 4) then $\langle C\rangle$ is a complete intersection. If $\langle A\rangle$ is not a complete intersection, then $I_{A}$ is generated by the $2 \times 2$ minors of a $2 \times 3$ matrix and, by Corollary [3.2, the minimal free resolution of $k[C]$ is as follows: $0 \rightarrow R^{2} \rightarrow R^{5} \rightarrow R^{4} \rightarrow R \rightarrow k[C] \rightarrow 0$.

At this point one can ask if every numerical semigroup of embedding dimension 4 that is an almost complete intersection of type 2 is decomposable. The answer is negative as the following example shows: the minimal free resolution of the semigroup $\langle C\rangle$ minimally generated by $C=\{9,11,13,15\}$ also has the following form, $0 \rightarrow R^{2} \rightarrow R^{5} \rightarrow R^{4} \rightarrow R \rightarrow$ $k[C] \rightarrow 0$. This semigroup is generated by an arithmetic sequence. In [12], the minimal graded free resolution of such semigroups has been completely described. In particular, one gets from [12, Thm. 4.1] that for every semigroup $\langle C\rangle$ of embedding dimension 4 generated by an arithmetic sequence, if the Cohen-Macaulay type of $k[C]$ is 2 then the minimal free resolution of $k[C]$ has the following form: $0 \rightarrow R^{2} \rightarrow R^{5} \rightarrow R^{4} \rightarrow R \rightarrow$ $k[C] \rightarrow 0$, and hence $\langle C\rangle$ is an almost complete intersection. These semigroups are all indecomposable by the following precise result: 
Proposition 5.2. A numerical semigroup minimally generated by a set of positive integers in arithmetic progression is decomposable if and only if it is of embedding dimension 3 and generated by a set of the form $\left\{2 c_{0}, 2 c_{0}+d, 2 c_{0}+2 d\right\}$ for $c_{0}$ and $d$ relatively prime and $d$ odd. In particular, it is indecomposable in embedding dimension 4 and higher.

Proof. If $C$ is an arithmetic sequence and $\langle C\rangle$ has embedding dimension $n$, then $C=$ $\left\{c_{0}+i d, 0 \leq i \leq n-1\right\}$ where $c_{0}$ and $d$ are relatively prime. If $C$ is decomposable as $k_{1} A \sqcup k_{2} B$, then $c_{0}$ and $c_{0}+d$ must be in different sets. Otherwise, they would have a common factor which would also be a common factor of $c_{0}$ and $d$. Similarly, $c_{0}+d$ and $c_{0}+2 d$ cannot be in the same set. Hence $c_{0}, c_{o}+2 d$ are in the same set, say $k_{1} A$. So $k_{1}$ divides $2 d$ and $c_{0}$, and hence $k_{1}=2$ and $c_{0}$ is even. Now, if $n \geq 4, c_{0}+d, c_{0}+3 d$ must be in $k_{2} B$ and hence $k_{2}$ divides $2 d$ and $c_{0}+d$. Since $c_{0}$ and $d$ are relatively prime, $k_{2}$ must also be 2 . Then all the integers in $C$ are even which is not possible. Thus, $C$ is indecomposable if $n \geq 4$. If $n=3, C$ must be of the form $\left\{2 c_{0}, 2 c_{0}+d, 2 c_{0}+2 d\right\}$ for $c_{0}$ and $d$ relatively prime and $d$ odd, which is the simple split $C=\left(2 c_{0}+d\right) \sqcup 2\left\{c_{0}, c_{0}+d\right\}$ : this is the only possibility for $\langle C\rangle$ to be decomposable if the elements in $C$ are in arithmetic progression.

Semigroups generated by arithmetic sequences provide examples of indecomposable semigroups of any type between 1 and the embedding dimension.

Corollary 5.3. For any $n \geq 4$ and any $t, 1 \leq t \leq n$, there exist indecomposable numerical semigroups of embedding dimension $n$ and type $t$.

Proof. This is a direct consequence of Proposition 5.2 and [12, Thm. 4.7].

We get, in particular, examples of Gorenstein semigroups that are indecomposable.

Example 6. For all $n \geq 3$, if $C=\left\{n+1,2 n+1,3 n+1, \ldots, n^{2}+1\right\}$ is a set of integers that minimally generates the numerical semigroup $\langle C\rangle$, the embedding dimension of $\langle C\rangle$ is $n$. For $n=3$, it fits into the family of decomposable semigroups given in Proposition 5.2 . For $n \geq 4$, it is a Gorenstein numerical semigroup by [12, Cor. 4.4] and it is indecomposable by Proposition [5.2. One can obtain all the Betti numbers of $k[C]$ using formula (4) in the proof of [12, Thm. 4.1]. In particular, the ideal $I_{C}$ is minimally generated by $(n+1)(n-2) / 2$ elements.

By Proposition 5.1, one gets that semigroups of embedding dimension 4 that are Gorenstein and not complete intersections are always indecomposable. For such a semigroup $\langle C\rangle$, Bresinsky showed in [4] that the ideal $I_{C}$ is minimally generated by 5 binomials of the form $\left\{x_{1}^{c_{1}}-x_{3}^{d_{13}} x_{4}^{d_{14}}, x_{3}^{c_{3}}-x_{1}^{d_{31}} x_{2}^{d_{32}}, x_{4}^{c_{4}}-x_{2}^{d_{42}} x_{3}^{d_{43}}, x_{2}^{c_{2}}-x_{1}^{d_{21}} x_{4}^{d_{24}}, x_{1}^{d_{21}} x_{3}^{d_{43}}-x_{2}^{d_{32}} x_{4}^{d_{14}}\right\}$. In [13], we give the structure of the minimal resolution of $k[C]$ in this case and explicitly write down the maps in the resolution as follows:

$$
0 \rightarrow R \stackrel{\delta_{3}}{\rightarrow} R^{5} \stackrel{\phi}{\rightarrow} R^{5} \stackrel{\delta_{1}}{\rightarrow} R \rightarrow k[C] \rightarrow 0
$$


where

$$
\phi=\left[\begin{array}{ccccc}
0 & 0 & x_{2}^{d_{32}} & x_{3}^{d_{43}} & x_{4}^{d_{24}} \\
0 & 0 & x_{1}^{d_{21}} & x_{4}^{d_{14}} & x_{2}^{d_{42}} \\
-x_{2}^{d_{32}} & -x_{1}^{d_{21}} & 0 & 0 & x_{3}^{d_{13}} \\
-x_{3}^{d_{43}} & -x_{4}^{d_{14}} & 0 & 0 & x_{1}^{d_{31}} \\
-x_{4}^{d_{24}} & -x_{2}^{d_{42}} & -x_{3}^{d_{13}} & -x_{1}^{d_{31}} & 0
\end{array}\right] \quad \text { and } \quad \delta_{3}=\left(\delta_{1}\right)^{T}=\left[\begin{array}{c}
x_{1}^{c_{1}}-x_{3}^{d_{13}} x_{4}^{d_{14}} \\
x_{3}^{c_{3}}-x_{1}^{d_{31}} x_{2}^{d_{32}} \\
x_{4}^{c_{4}}-x_{2}^{d_{42}} x_{3}^{d_{43}} \\
x_{2}^{c_{2}}-x_{1}^{d_{21}} x_{4}^{d_{24}} \\
x_{1}^{d_{21}} x_{3}^{d_{43}}-x_{2}^{d_{32}} x_{4}^{d_{14}}
\end{array}\right] .
$$

This was also obtained independently in [3]. Moreover, one can find in [13] a way to build families of numerical semigroups of embedding dimension 4 that are Gorenstein and not complete intersections.

For numerical semigroups of embedding dimension 5, one has the following result.

Proposition 5.4. In embedding dimension 5, the decomposable numerical semigroups are either complete intersections or type 2 almost complete intersections or obtained as a simple split $k_{1} \sqcup k_{2} A$ where $A$ is an indecomposable semigroup of embedding dimension 4.

Proof. If $\langle C\rangle$ is a simple split $C=k_{1} \sqcup k_{2} A$ then either $A$ is indecomposable, or $A$ is a complete intersection, or it is of type 2 and $I_{A}$ is minimally generated by 4 elements by Proposition 5.1. In the last case, by Theorem 4.1, $I_{C}$ is generated by 5 elements and it is of type 2 and hence it is an almost complete intersection of type 2 .

If $\langle C\rangle$ decomposes as $C=k_{1} A \sqcup k_{2} B$ where $A$ and $B$ have embedded dimension 2 and 3 respectively, if $B$ is indecomposable then by Theorem 3.1, $I_{C}$ is minimally generated by 5 elements and the type of $\langle C\rangle$ is 2 since it coincides with the type of $\langle B\rangle$. Again, in this case $\langle C\rangle$ is an almost complete intersection of type 2. If $B$ is decomposable, $\langle B\rangle$ is a complete intersection and so is $\langle C\rangle$.

In particular, one gets the following direct consequence.

Corollary 5.5. In embedding dimension 5, any decomposable Gorenstein numerical semigroup that is not a complete intersection must be obtained as simple split.

\section{Semigroups obtained By GLUing IN Higher Dimension}

One can now consider affine semigroups $\langle C\rangle$ finitely generated by a subset $C \subset \mathbb{N}^{n}$. As for numerical semigroups, given an arbitrary field $k$, if $C=\left\{\alpha_{1}, \ldots, \alpha_{s}\right\}$, one can consider the ring homomorfism $\phi: k\left[x_{1}, \ldots, x_{s}\right] \rightarrow k\left[t_{1}, \ldots, t_{n}\right]$ given by $\phi\left(x_{i}\right)=t^{\alpha_{i}}$ where for $\alpha=\left(a_{1}, \ldots, a_{n}\right) \in \mathbb{N}^{n}, t^{\alpha}=\prod_{j} t_{j}^{a_{j}}$. The kernel of $\phi$ is a binomial prime ideal, $I_{C}$, and the semigroup ring $k[C]$ is isomorphic to $k\left[x_{1}, \ldots, x_{s}\right] / I_{C}$. It is graded if one gives to each variable $x_{i}$ weight $\left|\alpha_{i}\right|$.

In this context, Rosales defined in [16] the concept of gluing. For an affine semigroup $\langle C\rangle$, when the set of generators of the semigroup splits into two disjoint parts, $C=A \sqcup B$, such that $I_{C}$ is minimally generated by $I_{A} \cup I_{B} \cup\{\rho\}$ where $\rho$ is a binomial whose first, respectively second, monomial involves only variables corresponding to elements in $A$, respectively $B$, we say that $\langle C\rangle$ is obtained by gluing $\langle A\rangle$ and $\langle B\rangle$. A characterization of 
this property in terms of the semigroups $\langle A\rangle$ and $\langle B\rangle$ and the groups associated to them is given in [16, Thm. 1.4]; see also [1].

In this situation, the argument in the proof of Theorem 3.1 is valid and hence Theorem 3.1 and Corollaries 3.2 and 3.3 hold. We will use again notations in section 2.2 except the ones involving $k_{1}$ and $k_{2}$ that are not defined in this general context.

Theorem 6.1. Consider an affine semigroup $\langle C\rangle$ obtained by gluing $\langle A\rangle$ and $\langle B\rangle$.

(1) $F_{A} \otimes F_{B}$ is a minimal graded free resolution of $R / I_{A} R+I_{B} R$.

(2) A minimal graded free resolution of the semigroup ring $k[C]$ can be obtained as the mapping cone of $\rho: F_{A} \otimes F_{B} \rightarrow F_{A} \otimes F_{B}$ where $\rho$ is induced by multiplication by $\rho$.

(3) The Betti numbers of $k[C]$ are

$\forall i \geq 0, \beta_{i}(C)=\sum_{i^{\prime}=0}^{i} \beta_{i^{\prime}}(A)\left[\beta_{i-i^{\prime}}(B)+\beta_{i-i^{\prime}-1}(B)\right]=\sum_{i^{\prime}=0}^{i} \beta_{i^{\prime}}(B)\left[\beta_{i-i^{\prime}}(A)+\beta_{i-i^{\prime}-1}(A)\right]$.

(4) In particular, the Cohen-Macaulay type of $k[C]$ is the product of the CohenMacaulay types of $k[A]$ and $k[B]$.

Proof. (11) and (2) are obtained exactly as Theorem 3.1 since our proof depends only on the form of the generating set of $I_{C}$. On the other hand, the proof of Corollary 3.2 also works in this general context by substituting $p-1$ and $q-1$ by $\operatorname{pd}(k[A])$ and $\operatorname{pd}(k[B])$, the projective dimensions of $k[A]$ and $k[B]$, and it gives (3). (4) is a direct consequence of (3).

A main difference between that case of numerical semigroups and the general case is that semigroup rings associated to affine semigroups are not always Cohen-Macaulay. The relation between the lengths of the minimal graded free resolutions of $k[A], k[B]$ and $k[C]$ is given by the following direct consequence of Theorem 6.1)(2):

Corollary 6.2. If the affine semigroup $\langle C\rangle$ is obtained by gluing $\langle A\rangle$ and $\langle B\rangle$, then $\operatorname{pd}(k[C])=\operatorname{pd}(k[A])+\operatorname{pd}(k[B])+1$.

Example 7. Consider the subsets of $\mathbb{N}^{3}, A=\{(4,0,0),(3,1,0),(2,2,0),(1,3,0)\}, B=$ $\{(3,3,0),(3,2,1),(3,1,2),(3,0,3)\}$ and $C=A \sqcup B$, and the affine semigroups $\langle A\rangle,\langle B\rangle$ and $\langle C\rangle$. Set $R_{A}=k\left[x_{1}, \ldots, x_{4}\right], R_{B}=k\left[y_{1}, \ldots, y_{4}\right]$ and $R=k\left[x_{1}, \ldots, y_{4}\right]$. Using Singular [8], one gets that the ideals $I_{A} \subset R_{A}$ and $I_{B} \subset R_{B}$ are minimally generated by $\left\{x_{3}^{2}-x_{2} x_{4}, x_{2}^{2}-x_{1} x_{3}, x_{2} x_{3}-x_{1} x_{4}\right\}$ and $\left\{y_{3}^{2}-y_{2} y_{4}, y_{2}^{2}-y_{1} y_{3}, y_{2} y_{3}-y_{1} y_{4}\right\}$, and the ideal $I_{C} \subset R$ is minimally generated by the union of those two sets and the element $\rho=y_{1}^{2}-x_{1} x_{4}^{4}$. The minimal free resolutions of the semigroup rings $k[A]$ and $k[B]$, as $R$-modules, are of the following form, $0 \rightarrow R^{2} \rightarrow R^{3} \rightarrow R$ and, by Theorem 6.1, that of $k[C]$ is $0 \rightarrow R^{4} \rightarrow R^{16} \rightarrow R^{25} \rightarrow R^{19} \rightarrow R^{7} \rightarrow R$.

\section{ACKNOWLEDGEMENTS}

The second author acknowleges with pleasure the support and hospitality of University of Valladolid while working on this project. 


\section{REFERENCES}

[1] A. Assi, P. A. García-Sánchez and I. Ojeda, Frobenius vectors, Hilbert series and gluings of affine semigroups, J. Commut. Algebra 7 (2015), 317-335.

[2] L. L. Avramov, Infinite free resolutions. In: Six lectures on commutative algebra (Bellaterra, 1996), Prog. Math. 166, Birkhäuser, 1998, 1-118.

[3] V. Barucci, R. Fröberg and M. Şahin, On free resolutions of some semigroup rings, J. Pure Appl. Algebra 218 (2014), 1107-1116.

[4] H. Bresinsky, Symmetric semigroups of integers generated by 4 elements, Manuscr. Math. 17 (1975), $205-219$

[5] D.A. Buchsbaum and D. Eisenbud, What makes a complex exact, J. Algebra 25 (1973), $259-268$.

[6] D.A. Buchsbaum and D. Eisenbud, Algebra structures for finite free resolutions and some structure theorems for ideals of codimension 3, American J. Math. 99 (1977), 447-485.

[7] A. Campillo and P. Gimenez, Syzygies of affine toric varieties, J. Algebra 225 (2000), 142-161.

[8] W. Decker, G.-M. Greuel, G. Pfister and H. Schönemann, Singular 4-1-1 - A computer algebra system for polynomial computations, 2018. www.singular.uni-kl.de.

[9] C. Delorme, Sous-monoïdes d'intersection complète de N, Ann. Sci. Éc. Norm. Sup. (4) 9 (1976), $145-154$.

[10] D. Eisenbud, The geometry of syzygies, Graded Texts in Mathematics 229, Springer, 2005.

[11] R. Fröberg, C. Gottlieb and R. Häggkvist On numerical semigroup, Semigroup Forum 35 (1987), $63-83$.

[12] P. Gimenez, I. Sengupta and H. Srinivasan, Minimal graded free resolutions for monomial curves defined by arithmetic sequences, J. Algebra 388 (2013), 294-310.

[13] P. Gimenez and H. Srinivasan, A note on Gorenstein monomial curves, Bull. Braz. Math. Soc. New Series 45 (2014), 671-678.

[14] J. Herzog, Generators and relations of abelian semigroups and semigroup rings, Manuscr. Math. 3 (1970), 175-193.

[15] H. Nari, Symmetries on almost arithmetic numerical semigroups, Semigroup Forum 86 (2013), 140154.

[16] J. C. Rosales, On presentations of subsemigroups of $\mathbb{N}^{n}$, Semigroup Forum 55 (1997), $152-159$.

[17] J. C. Rosales and P. A. García-Sánchez, Numerical Semigroups, Developments in Mathematics 20, Springer, 2009.

[18] H. Srinivasan, Ranks of syzygies of perfect modules, J. Pure Appl. Algebra 65 (1990), 91-96.

Instituto de Matemáticas de la Universidad de Valladolid (IMUVA), Facultad de CienCIAS, 47011 VAlladolid, SPAin.

E-mail address: pgimenez@agt.uva.es

Mathematics Department, University of Missouri, Columbia, MO 65211, USA.

E-mail address: SrinivasanH@math.missouri.edu 